\title{
SOME RESULTS ON THE COMPLEX OSCILLATION THEORY OF SECOND ORDER LINEAR DIFFERENTIAL EQUATIONS
}

\author{
By Cun-Zhi HuANG
}

\section{Introduction}

We consider the second order linear differential equation

$$
f^{\prime \prime}+A f=0,
$$

where $A$ is an entire function. For an entire function $f$, let $\rho(f)$ be its order, $\mu(f)$ its lower order and $\lambda(f)$ the exponent of convergence of its zeros. In addition, we assume that the reader is familiar with the standard notations of Nevanlinna theory (see [3]).

When $A$ is a polynomial of degree $n \geqq 1$, S. Bank and I. Laine obtained the following ([1]).

ThEOREM A. Let $A$ be a polynomial of degree $n \geqq 1$. If $f \neq 0$ is a solution of (1), then

$$
\rho(f)=(n+2) / 2,
$$

and if $f_{1}, f_{2}$ are two linearly independent solutions of (1), then

$$
\max \left(\lambda\left(f_{1}\right), \lambda\left(f_{2}\right)\right)=(n+2) / 2 \text {. }
$$

If $A$ is transcendental, we apply the lemma on the logarithmic derivative in Nevanlinna theory to (1) and can deduce that any solution $f \neq 0$ of (1) satisfies

We may hope that

$$
\rho(f)=\infty \text {. }
$$

$$
\max \left(\lambda\left(f_{1}\right), \lambda\left(f_{2}\right)\right)=\infty,
$$

where $f_{1}$ and $f_{2}$ are linearly independent solutions of (1). However, examples in [1] show that this is not the case. Specifically, for $\rho(A)$ a positive integer or infinity, there exist $A$ and independent solutions $f_{1}, f_{2}$ of (1) such that

Received August 30, 1990. 


$$
\max \left(\lambda\left(f_{1}\right), \lambda\left(f_{2}\right)\right)<\infty .
$$

When the growth of $A$ is suitably restricted, the following were obtained.

THEOREM B ([6]). Let $A$ be a transcendental entire function of finite order and of lower order $\mu \leqq 1 / 2$. If $f_{1}$ and $f_{2}$ are two linearly independent solutions of (1), then

$$
\max \left(\lambda\left(f_{1}\right), \lambda\left(f_{2}\right)\right)=\infty \text {. }
$$

Theorem C ([5]). Let $A$ be a transcendental entire function of order $\rho(A)<1$. If $f_{1}$ and $f_{2}$ are two linearly independent solutions of (1), then

or

$$
\lambda\left(f_{1} f_{2}\right)=\infty
$$

$$
\rho(A)^{-1}+\lambda\left(f_{1} f_{2}\right)^{-1} \leqq 2 .
$$

In this paper, we prove

THEOREM 1. Let $A$ be a transcendental entire function of lower order $\mu(A)<1$. If $f_{1}$ and $f_{2}$ are two linearly independent solutions of $(1)$, then

or

$$
\lambda\left(f_{1} f_{2}\right)=\infty
$$

$$
\mu(A)^{-1}+\lambda\left(f_{1} f_{2}\right)^{-1} \leqq 2 .
$$

Remark. Theorem 1 generalizes Theorem B and Theorem C. Furthermore, we note that the condition $\rho(A)$ is finite in Theorem $\mathrm{B}$ is not necessary.

Before stating Theorem 2, we introduce some definitions. Let

$$
f(z)=\sum_{n=0}^{\infty} a_{n} z^{n}
$$

be a transcendental entire function. We denote by $\Lambda=\left\{\lambda_{k}\right\}, M=\left\{\mu_{k}\right\}(k=1,2, \cdots)$ the sequences of exponents $n$ for which $a_{n} \neq 0$ and $a_{n}=0$ respectively, arranged in increasing order. We say that $f(z)$ has Fabry gap if

$$
\frac{\lambda n}{n} \longrightarrow \infty \quad(n \rightarrow \infty)
$$

Now we have

THEOREM 2. Let $A$ be a transcendental entire function of lower order $\mu(A)<\infty$ and have Fabry gap. If $f_{1}$ and $f_{2}$ are two linearly independent solutions of (1), then

$$
\max \left(\lambda\left(f_{1}\right), \lambda\left(f_{2}\right)\right)=\infty
$$




\section{Preliminaries}

LEMMA 1. Let $E$ be an entire function of finite order, then there exist a positive integer $q$ and $a$ set $\Delta \subset[1, \infty)$ of finite linear measure, such that for $z \bar{\epsilon} \Delta^{*}$, we have

$$
2\left|E^{\prime \prime} / E(z)\right|+\left|E^{\prime} / E(z)\right|^{2}<|z|^{q},
$$

where $\Delta^{*}=\{z:|z| \in \Delta\}$.

This lemma can be deduced from [2].

From [4, Theorem 4], we have

LEMma 2. Suppose that $A(z)$ is an entire function and has Fabry gap such that for some arbitrarily large $R$ we have

$$
\log M(R, A)<R^{\lambda},
$$

where $\lambda$ is a positive constant. Let $\eta_{1}, \eta_{2}$ be constants between 0 and 1 , then there exists a subset $\boldsymbol{E}$ of the real axis, such that the logarithmic measure of $\boldsymbol{E} \cap[1, R]$ is at least $\left(1-\eta_{1}\right) \log R+O(1)$, as $R \rightarrow \infty$ through values satisfying (6) and such that we have, for $r$ in $\boldsymbol{E}$,

$$
\log L(r, A)>\left(1-\eta_{2}\right) \log M(r, A),
$$

where $L(r, A)=\min _{|z|=r}|A(z)|$ and $M(r, A)=\max _{|z|=r}|A(z)|$.

\section{Proof of Theorem 1}

Let $f_{1}$ and $f_{2}$ be two linearly independent solutions of (1). Set $E=f_{1} f_{2}$, then we note as in [1] that

$$
-4 A=(c / E)^{2}-\left(E^{\prime} / E\right)^{2}+2\left(E^{\prime \prime} / E\right),
$$

where $c$ is the constant Wronskian of $f_{1}$ and $f_{2}$. Applying Nevanlinna theory to (7), we have

$$
T(r, E)=N(r, 1 / E)+\frac{1}{2} T(r, A)+O(\log (r T(r, E)))
$$

as $r \rightarrow \infty$ outside a set of finite measure.

We assume that $\rho(E)<\infty$. Since $\mu(A)<1, A(z)$ must have infinitely many zeros. Let $a_{1}, a_{2}, \cdots, a_{q+1}$ be $q+1$ zeros of $A(z)$ with $q$ as in Lemma 1 . Define

$$
H(z)=A(z) / \prod_{\imath=1}^{q+1}\left(z-a_{\imath}\right)
$$

then $H$ is entire and of lower order $\mu(H)=\mu(A)<1$.

Set 


$$
\begin{aligned}
& D(H)=\{z:|H(z)|>1\}, \\
& D(E)=\{z:|E(z)|>1\} .
\end{aligned}
$$

Since $H(z)$ and $E(z)$ are transcendental, there exist $z_{i} \in C(i=1,2)$ such that

$$
\begin{aligned}
& \left|H\left(z_{1}\right)\right|>e, \\
& \left|E\left(z_{2}\right)\right|>e .
\end{aligned}
$$

Let $\Omega_{1}\left(\Omega_{2}\right)$ be the connected component of $D(H)(D(E))$ containing the point $z_{1}\left(z_{2}\right)$. By the maximum modulus principle, we conclude that $\Omega_{i}(i=1,2)$ are unbounded.

Set

$$
r_{0}=\max \left\{1,\left|z_{1}\right|,\left|z_{2}\right|\right\} .
$$

Let $\theta_{i t}\left(i=1,2 ; r_{0} \leqq t<\infty\right)$ be the part of the circle $|z|=t$ in $\Omega_{\imath}$ and $t \theta_{i}(t)$ the linear measure of $\theta_{i t}$.

By Lemma 1 and (7), we deduce that

$$
4|A(z)| \leqq|c|^{2}+|z|^{q}, \quad z \in D(E)-\Delta^{*} .
$$

But for $z \in D(H)-\Delta^{*}$ and $|z|$ sufficiently large, we have

$$
|A(z)| \geqq \frac{1}{2}|z|^{q+1} \text {. }
$$

From (9) and (10), we have for $r_{0}^{\prime}$ large enough

(11) implies

$$
\left(D(E)-\Delta^{*}\right) \cap\left(D(H)-\Delta^{*}\right) \cap\left\{z:|z|>r_{0}{ }^{\prime}\right\}=\phi .
$$

$$
\theta_{1}(t)+\theta_{2}(t) \leqq 2 \pi, \quad t \in \Delta .
$$

By a theorem of Tsuji [7], we have

$$
\log \left|H\left(z_{1}\right)\right| \leqq 9 \sqrt{2} \exp \left(-\pi \int_{2 r_{0}}^{(1 / 2) r} \frac{d t}{t \theta_{1}(t)}\right) \log M(r, H) .
$$

(13) gives

$$
\pi \int_{2 r_{0}}^{(1 / 2) r} \frac{d t}{t \theta_{1}(t)} \leqq \log \log M(r, H)+\log (9 \sqrt{2}) .
$$

Set

$$
G(r)=\left[2 r_{0}, \frac{1}{2} r\right]-\Delta
$$

and

$$
\alpha=\varliminf_{r \rightarrow \infty}(\log r)^{-1} \pi \int_{G(r)} \frac{d t}{t \theta_{1}(t)},
$$

then, from (14), we have 


$$
\frac{1}{2} \leqq \alpha \leqq \mu(H) .
$$

Similarly

$$
\pi \int_{G(r)} \frac{d t}{t \theta_{2}(t)} \leqq \log \log M(r, E)+\log (9 \sqrt{2}) .
$$

By Cauchy-Schwarz inequality

$$
\int_{G(r)} \frac{\theta_{i}(t)}{t} d t \int_{G(r)} \frac{d t}{t \theta_{i}(t)} \geqq\left(\int_{G(r)} \frac{d t}{t}\right)^{2}, \quad i=1,2 .
$$

From (12) and (17) with $i=1$, we obtain

$$
\begin{aligned}
\int_{G(r)} \frac{\theta_{2}(t)}{t} d t & \leqq \int_{G(r)} \frac{2 \pi-\theta_{1}(t)}{t} d t \\
& =2 \pi \int_{G(r)} \frac{d t}{t}-\int_{G(r)} \frac{\theta_{1}(t)}{t} d t \\
& \leqq 2 \pi \int_{G(r)} \frac{d t}{t}-\frac{\left(\int_{G(r)} \frac{d t}{t}\right)^{2}}{\int_{G(r)} \frac{d t}{t \theta_{1}(t)}}
\end{aligned}
$$

(18) and (17) with $i=2$ give

$$
\begin{aligned}
\int_{G(r)} \frac{d t}{t \theta_{2}(t)} & \geqq \frac{\left(\int_{G(r)} \frac{d t}{t}\right)^{2}}{2 \pi \int_{G(r)} \frac{d t}{t}-\frac{\left(\int_{G(r)} \frac{d t}{t}\right)^{2}}{\int_{G(r)} \frac{d t}{t \theta_{1}(t)}}} \\
& =\frac{\int_{G(r)} \frac{d t}{t}}{2 \pi-\frac{\int_{G(r)} \frac{d t}{t}}{\int_{G(r)} \frac{d t}{t \theta_{1}(t)}}} .
\end{aligned}
$$

Since $\Delta$ is of finite linear measure, from (16) and (19), we obtain

$$
\begin{aligned}
\rho(E) & =\varlimsup_{r \rightarrow \infty} \frac{\log \log M(r, E)}{\log r} \\
& \geqq \varlimsup_{r \rightarrow \infty}(\log r)^{-1} \pi \int_{G(r)} \frac{d t}{t \theta_{2}(t)} \\
& \geqq \frac{1}{2-\frac{1}{\alpha}} \\
& =\frac{\alpha}{2 \alpha-1} .
\end{aligned}
$$


Inequalities (15) and (20) give

which implies

$$
\rho(E) \geqq \frac{\mu(H)}{2 \mu(H)-1},
$$

$$
\rho(E)^{-1}+\mu(A)^{-1} \leqq 2 .
$$

We assert that $\rho(E)=\infty$ implies $\lambda(E)=\infty$. If $\lambda(E)<\infty$, we would arrive at a contradiction.

Let $E=P e^{Q}$ where $P$ is a canonical product formed by the zeros of $E$ and $Q$ is an entire function, then $\rho(P)<\infty$. Since $\rho(E)=\infty, Q$ must be transcendental. From here, we conclude that $\mu(E)=\infty$.

For any $\alpha>1$, we have by (8)

$$
\frac{1}{2} T(r, E) \leqq N(\alpha r, 1 / E)+\frac{1}{2} T(\alpha r, A), \quad(r \text { large enough }) .
$$

(22), $\mu(A)<1$ and $\lambda(E)<\infty$ imply

$$
\mu(E)<\infty .
$$

This gives a contradiction.

Similarly, we can prove that if $\rho(E)<\infty$, then $\lambda(E)=\rho(E)$. From (21), we have

$$
\lambda(E)^{-1}+\mu(A)^{-1} \leqq 2 .
$$

We have completed the proof of Theorem 1.

\section{Proof of Theorem 2}

Applying the Wiman-Valiron theory to (7), we conclude that there exists a set $D$ in $[1, \infty)$ of finite logarithmic measure such that if $r \in D$ and $z$ is a point on $|z|=r$ at which $|E(z)|=M(r, E)$, then

$$
2|A(z)| \leqq\left(\frac{\nu(r)}{r}\right)^{2}
$$

where $\nu(r)$ denotes the central index of $E$. It follows from Lemma 2 that there exists a sequence $r_{n} \rightarrow \infty$ such that $r_{n} \in \boldsymbol{E}-D$

$$
L\left(r_{n}, A\right) \leqq M\left(r_{n}, A\right)^{1 / 2} .
$$

From (23) and (24), we have for any positive integer $N$

$$
r_{n}{ }^{N} \leqq \frac{\nu\left(r_{n}\right)}{r_{n}},
$$

which implies

$$
\rho(E)=\infty
$$


The proof of Theorem 2 can be completed in the same way as that of Theorem 1 .

\section{REFERENCES}

[1] S. BANK AND I. LAINe, On the oscillation theory of $f^{\prime \prime}+A f=0$ where A is entire, Trans. Amer. Math. Soc. 273 (1982), 351-363.

[2] G. G. Gundersen, Estimates for the logarithmic derivative of a meromorphic function, plus similar estimates, J. London Math. Soc. (1) 37 (1988), 88-104.

[3] W.K. Hayman, Meromorphic functions, University Press, Oxford (1964).

[4] W.K. Hayman, Angular value distribution of power series with gaps, Proc. London Math. Soc. (3) 24 (1972), 590-624.

[5] J. Rossi, Second order differential equations with transcendental coefficients, Proc. Amer. Math. Soc. 97 (1986), 61-66.

[6] L.C. SHEN, Solution to a problem of S. Bank regarding exponent of convergence of zeros of the solutions of differential equation $f^{\prime \prime}+A f=0$, Kexue Tongbao (Chinese) 30 (1985), 1579-1585.

[7] M. TsujI, On Borel's directions of meromorphic functions of finite order. I, Tôhoku Math. J. 2 (1950), 97-112.

Department of Mathematics

East China Normal University

SHANGHAI 200062

P. R. CHINA 